\title{
Infection in a rat model reactivates attenuated virulence after long-term axenic culture of Acanthamoeba spp
}

\author{
Carolina De Marco Veríssimoํㅜ, Vinícius José Maschio', \\ Ana Paula Folmer Correa ${ }^{2}$, Adriano Brandelli², Marilise Brittes Rott ${ }^{3 /+}$
}

'Laboratório de Parasitologia ${ }^{3}$ Departamento de Microbiologia, Setor de Parasitologia, Instituto de Ciências Básicas da Saúde ${ }^{2}$ Laboratório de Bioquímica e Microbiologia Aplicada, Instituto de Ciência e Tecnologia dos Alimentos, Universidade Federal do Rio Grande do Sul, Porto Alegre, RS, Brasil

Prolonged culturing of many microorganisms leads to the loss of virulence and a reduction of their infective capacity. However, little is known about the changes in the pathogenic strains of Acanthamoeba after long culture periods. Our study evaluated the effect of prolonged culturing on the invasiveness of different isolates of Acanthamoeba in an in vivo rat model. ATCC strains of Acanthamoeba, isolates from the environment and clinical cases were evaluated. The in vivo model was effective in establishing the infection and differentiating the pathogenicity of the isolates and re-isolates. The amoebae cultured in the laboratory for long periods were less virulent than those that were recently isolated, confirming the importance of passing Acanthamoeba strains in animal models.

Key words: Acanthamoeba - virulence - in vivo

Acanthamoeba, a genus of free-living amoebae, is widely dispersed in the environment and can be found in natural water bodies, dust, air conditioners, swimming pools and hospitals (Marciano-Cabral \& Cabral 2003, Caumo et al. 2009, Magliano et al. 2009, Carlesso et al. 2010). The clinical importance of these organisms is related to their ability to cause infections, such as amoebic keratitis, which affects healthy individuals, particularly users of contact lenses. In rare cases, Acanthamoeba is the agent responsible for granulomatous amoebic encephalitis (GAE), which is a progressive infection of the central nervous system (CNS) that almost exclusively affects immunocompromised individuals. GAE is characterised by an intense inflammatory response, the invasion of the blood-brain barrier and neurological damage progressing to brain dysfunction, ultimately leading to the death of the patient within days or weeks (Martinez \& Visvesvara 1997, Marciano-Cabral \& Cabral 2003, Khan 2006).

GAE is initiated by the penetration of the protist intranasally or through a skin lesion (Martinez 1985, 1991, Martinez \& Visvesvara 1997). Being opportunistic agents, Acanthamoeba can invade and colonise other tissues, causing disseminated infections that affect the lungs, liver, kidneys, skin, pancreas and prostate, suggesting that the spread of these agents throughout the body occurs haemotogenously (Khan 2006, Khan \& Siddiqui 2009, Mortazavi et al. 2009).

\footnotetext{
doi: 10.1590/0074-0276130099

Financial support: $\mathrm{CNPq}$

+ Corresponding author: marilise.rott@ufrgs.br

Received 3 November 2012

Accepted 26 March 2013
}

Research on the pathogenic potential of Acanthamoe$b a$ is complicated by the changes induced by prolonged axenic laboratory culture, such as the downregulation of its virulence and encystment capacity and its altered sensitivity to drugs (Mazur \& Hadas 1994, Hughes et al. 2003, Koehsler et al. 2008). These adaptations clearly affect studies on the pathogenic potential and drug susceptibility of Acanthamoeba and an effective method to maintain or reactivate the properties of freshly isolated amoebae is highly desirable.

A decrease in the virulence of Acanthamoeba is not permanent and can be restored by multiple mouse passages (Mazur \& Hadas 1994). The present study evaluated the effect of the prolonged culturing of different isolates of Acanthamoeba on their invasiveness in a rat model.

\section{MATERIALS AND METHODS}

Acanthamoeba isolates and cultivation - Clinical and environmental strains of Acanthamoeba polyphaga (AP2 - ATCC 30461 and AP4 - ATCC 30872) and Acanthamoeba castellanii (T4 - ATCC 50492 and NEFF - ATCC 30010) were used in this study. The organisms were grown at $30^{\circ} \mathrm{C}$ in $2 \%$ proteose peptone, $0.2 \%$ yeast extract and $1.8 \%$ glucose containing antibiotics (penicillin and streptomycin $40 \mu \mathrm{L} / \mathrm{mL}$ ) to maintain axenic culture. The ATCC strains used have been grown in this manner in the laboratory since 2006.

In vivo infection model of Acanthamoeba using intranasal inoculation - The stimulant solution (SS) for infection was prepared from cultures of different isolates of Acanthamoeba. The medium containing $1 \times 10^{6}$ trophozoites $/ \mathrm{mL}$ was centrifuged at $250 \mathrm{~g}$ for $10 \mathrm{~min}$. The pellet was washed three times with phosphate buffered saline (PBS) and resuspended in $200 \mu \mathrm{L}$ of PBS. Adult male Wistar rats, with a mean age of three months, were first immunosuppressed by intraperitoneal injection with dexamethasone (5 mg/kg) (Cappila et al. 2006). For 
infection, the animals were anesthetised with ketamine hydrochloride $(60 \mathrm{mg} / \mathrm{kg})$ and xylazine hydrochloride $(8$ $\mathrm{mg} / \mathrm{kg}$ ) and were inoculated intranasally with $200 \mu \mathrm{L}$ of the SS. After infection, the animals were observed for 30 days. After this period, the rats were euthanised by depression of oxygen and the organs were removed and evaluated under a magnifying glass to observe the lesions. All experiments were performed in duplicate. Two animals were used as controls and were submitted to the same conditions as the test group. The parasites were re-isolated from the organ lesions and cultured in plates with non-nutrient agar $1.5 \%$ covered with inactivated Escherichia coli (ATCC 25922). Only the T4r and AP4r re-isolates were re-inoculated into animals.

Ethics - This study had been previously approved by the Ethical Committee on Animal Studies at the Federal University of Rio Grande do Sul.

Histological analysis - The organs were removed from the infected animals and preserved in 4\% paraformaldehyde. Later, sections were obtained for histological analysis and protist visualisation in tissue. Haematoxylin and eosin were used to stain the sections.

\section{RESULTS}

All the ATCC isolates studied, i.e., T4 (50492), AP2 (30461), AP4 (30872) and NEFF (30010), were able to establish systemic infections in the rats. The lesions produced by AP2 (30461) and NEFF (30010) were disseminated in several organs and caused the deaths of the animals after an average period of 26 days. The lesions produced by AP4 (30872) and T4 (50492) were restricted to the lungs and these animals survived for the 30-day duration of the experiment (Table). For the rats infected with $\mathrm{T} 4 \mathrm{r}$ and $\mathrm{AP} 4 \mathrm{r}$, the mean survival time after infection was 17 days (Table). Generally, it can be assumed that the rats survived for shorter periods when the infections were more invasive. The two animals used as controls survived the 30-day period of the experiment and post-mortem examination revealed no injury to their organs. Acanthamoeba were not isolated from the tissues obtained from the control rats.
All of the Acanthamoeba isolates, whether clinical or environmental, were able to establish systemic infections in the immunosuppressed animals, where they mainly reached the lungs, as shown in Fig. 1. The animals infected with the standard strains, i.e., AP2 (30461) and NEFF (30010), suffered more invasive infections. The parasite was re-isolated from the lungs, liver and kidneys of rats infected with the ATCC strain 30461, as well as from the liver and lungs of rats infected with the ATCC strain 30010 (Table). When the trophozoites that were re-isolated from lesions were used, i.e., AP4r and $\mathrm{T} 4 \mathrm{r}$, the protists were found in several organs, including the brain (Table). The symptoms were perceived 10 days after infection and mainly consisted of weight loss, hair loss, shivering, diarrhoea and prostration.

The brains of all the infected animals were removed and observed. Only the brains of animals infected with re-isolated $\mathrm{AP} 4 \mathrm{r}$ and $\mathrm{T} 4 \mathrm{r}$ showed altered morphology (Fig. 2). The parasites were observable in the histological analyses (Fig. 2).

\section{DISCUSSION}

During human infection, strains of Acanthamoeba affect several organs in addition to the brain, including the skin, liver, lungs, kidneys, heart, diaphragm, eyes, adrenal glands, pancreas, prostate, lymph nodes and bone marrow (Mazur \& Jóźwiak 1993, Khan 2010, Young et al. 2010). Mortazavi et al. (2009) studied Acanthamoeba infections in locusts and observed that the insects developed disseminated infections in the haemolymph, fat body, muscle and brain tissues. In another study, Acanthamoeba were found in the lungs and brain tissue of mice (de Jonckheere 1980). The results of the present study showed that this infection model produced systemic infections in rats and that several organs were affected (Table).

According to Khan (2010), the ability of Acanthamoe$b a$ to cause systemic infections reflects its capacity to survive, attack the innate immune system and invade the CNS, which is consistent with GAE cases in humans. Our results show that strains isolated from the environment can cause infections that are quite similar to those caused by the clinical strains of Acanthamoeba (Table), which

TABLE

Evaluation of in vivo model of Acanthamoeba infection

\begin{tabular}{lcccccc}
\hline & \multicolumn{5}{c}{ Isolation in organs } \\
Isolated & Lungs & Brain & Liver & Heart & Kidneys & $\begin{array}{c}\text { Days of survival } \\
\text { post-infection }\end{array}$ \\
\hline \cline { 2 - 6 } T4 & + & - & - & - & - & 30 \\
AP2 & + & - & + & - & + & 22 \\
AP4 & + & - & - & - & - & 30 \\
NEFF & + & + & - & + & - & 30 \\
T4r & + & + & + & + & + & 20 \\
AP4r & + & & & & + & 15 \\
\hline
\end{tabular}

AP2: ATCC 30461; AP4: ATCC 30872; NEFF: ATCC 30010; T4: ATCC 50492. 
poses a potential risk to human health, especially in immunosuppressed individuals. This risk is consistent with the results of de Jonckheere (1980), who found that strains previously described as incapable of causing infections in mice were highly pathogenic and caused $100 \%$ mortality in animals that were inoculated intracerebrally.

When recent isolates of Acanthamoeba were used to infect rats, the animals developed more invasive and rapidly fatal infections. For Leishmania spp, maintenance in vitro results in a progressive loss of virulence, which can be reversed by passage in a mammalian host. A significant correlation has also been found between higher parasite burdens in infected Balb/c mice and fewer in vitro passages of the amoebae (Moreira et al. 2012). Therefore, the different invasive capacities of Acanthamoeba isolates and re-isolates can be explained by the expression of different virulence factors after the first infection.
Mazur et al. (1999) compared the pathological and morphological changes in the organs of rats caused by infection with strains of low and high virulence in vivo. These investigators reported that infections with each Acanthamoeba isolate resulted in similar infections, but that the changes induced by the strain with low virulence developed later and were less extensive. They suggested that differences in the virulence of strains, due to the reactivation of factors, could explain why only the reisolated amoebae reached the brain of the rats. Another study demonstrated that parasites that had been grown for different periods of axenic in vitro culture induced different infections in rats (Moreira et al. 2012).

Studies in the 1970s demonstrated that when studying Acanthamoeba, pathogenicity tests should be performed as soon as possible after isolation because the strains lose virulence with frequent transfers in axenic culture (Ste-
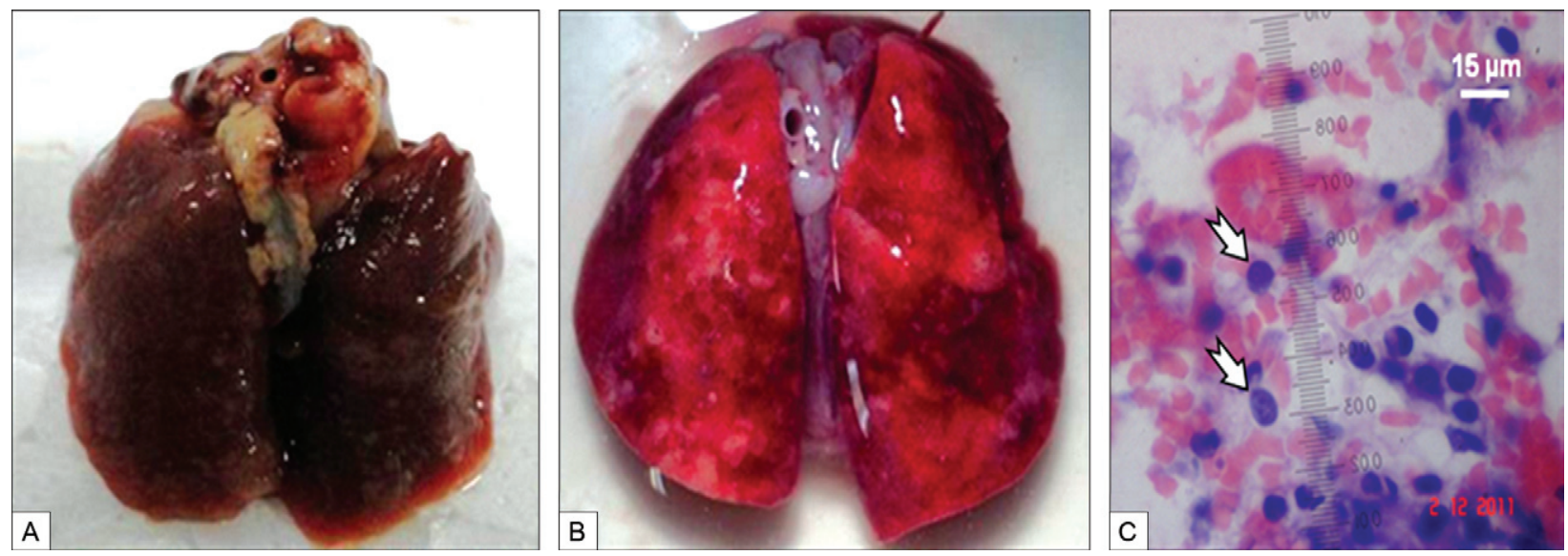

Fig. 1: representative pictures of lungs of rats infected intranasally with Acanthamoeba. A: lungs normal tissue, rats not infected with Acanthamoeba; B: lungs tissue with confirmed Acanthamoeba infection; C: histological analysis of tissue the lungs with Acanthamoeba contamination. The arrows point to two trophozoites. Magnification 400X.
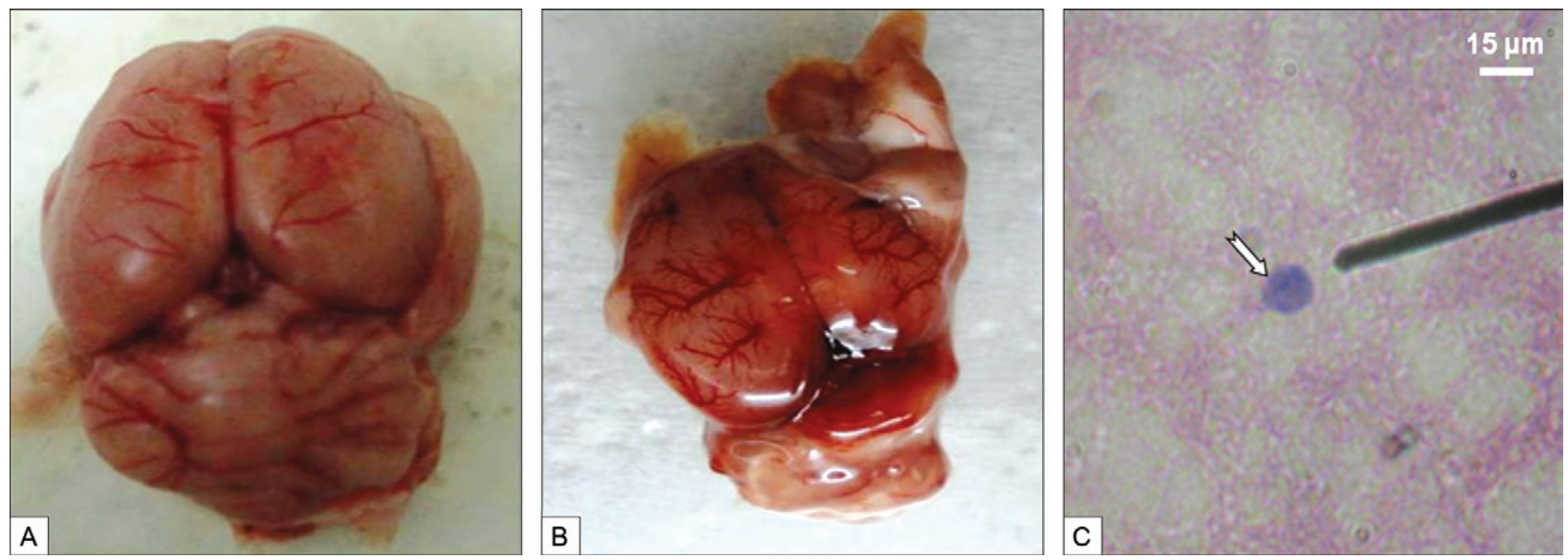

Fig. 2: representative pictures of brain of rats infected intranasally with Acanthamoeba. A: brain normal tissue, rats not infected with Acanthamoeba; B: brain tissue with confirmed Acanthamoeba infection (T4r re-isolate); C: histological analysis of tissue the brain with Acanthamoe$b a$ contamination. The arrows point the trophozoite. Magnification 400X. 
vens \& O’Dell 1974). Moreira et al. (2012) noted that axenic culturing and long-term maintenance of the parasite can affect the outcome of in vitro infections. This influence was also demonstrated in in vivo studies by Górnik and Kuźna-Grygiel (2005), who determined the degrees of virulence for six strains of Acanthamoeba in mice based on the number of organs affected and the extent of histopathological changes due to systemic infections. Using this criterion, the strains studied here were classified with varying degrees of virulence. As such, ATCC strains AP2 (30461) and NEFF (30010) were more virulent and T4 (30492) and AP4 (30872) were less virulent. Additionally, the T4r and AP4r re-isolates became more virulent after the first infection in animals.

Although in vitro models are more practical and efficient in restoring the virulence of Acanthamoeba (Koehsler et al. 2009), this study utilised an in vivo model because, even with immunosuppressed animals, the parasites must address different host defence mechanisms, which can result in the expression of different virulence factors and the selection of resistant forms. The capacity of the parasite to modulate macrophage activation decreases significantly with increasing numbers or intervals of in vitro passages (Borba 2002, Santos et al. 2007, Moreira et al. 2012).

The observed changes in the profiles of proteolytic activity between the isolates cultured in the laboratory for long periods and those recently passed through an animal model (data not shown), as well as the increase in the severity of the infections caused by these re-isolates, indicate that the first contact with animals may have induced changes in the expression of the virulence factors.

Our results provide further evidence for the effect of prolonged axenic culture on the virulence of Acanthamoeba. They indicate the importance of developing protocols to reactivate the virulence of Acanthamoeba cultures in studies focusing on interactions with mammals, such as drug development or vaccine trial studies and pathogenicity.

\section{ACKNOWLEDGEMENTS}

To Elle Ditmer, who reviewed the English.

\section{REFERENCES}

Borba CM 2002. Análise morfológica, bioquímica e molecular de cepas de Paracoccidioides brasiliensis exibindo diferenças na patogenicidade, $\mathrm{PhD}$ Thesis, Fiocruz, Rio de Janeiro, $96 \mathrm{pp}$.

Cappila J, Maffei CM, Clemons KV 2006. Experimental systemic infection with Cryptococcus neoformans var. and Cryptococcus gat$t i$ in normal and immunodeficient mice. Med Mycol 44: 601-610.

Carlesso AM, Artuso GL, Caumo K, Rott MB 2010. Potentially pathogenic Acanthamoeba isolated from a hospital in Brazil. Curr Microbiol 60: 185-190.

Caumo K, Frasson AP, Pens CJ, Panatieri LF, Razzon APG, Rott MB 2009. Potentially pathogenic Acanthamoeba in swimming pools: a survey in the southern Brazilian city of Porto Alegre. Ann Trop Med Parasitol 103: 477-485.

de Jonckheere JF 1980. Growth characteristics, cytopathic effect in cell culture and virulence in mice of 36 type strains belonging to 19 different Acanthamoeba spp. Appl Environ Microbiol 39: 681-685.

Górnik K, Kuźna-Grygiel W 2005. Histological studies of selected organs of mice experimentally infected with Acanthamoeba spp. Folia Morphol (Warsz) 64: 161-167.
Hughes R, Heaselgrave W, Kilvington S 2003. Acanthamoeba polyphaga strain age and method of cyst production influence the observed efficacy of therapeutic agents and contact lens disinfectants. Antimicrob Agents Chemother 47: 3080-3084.

Khan NA 2006. Acanthamoeba: biology and increasing importance in human health. FEMS Microbiol Rev 30: 564-595.

Khan NA 2010. Novel in vitro and in vivo models to study central nervous system infections due to Acanthamoeba spp. Exp Parasitol 126: 69-72.

Khan NA, Siddiqui R 2009. Acanthamoeba affects the integrity of human brain microvascular endothelial cells and degrades the tight junction proteins. Int J Parasitol 3: 1611-1616.

Koehsler M, Leitsch D, Duchene M, Nagl M, Walochnik J 2009. Acanthamoeba castellanii: growth of human cell layer reactivates attenuated properties after prolonged axenic culture. FEMS Microbiol Lett 299: 121-127.

Koehsler M, Leitsch D, Fuernkranz U, Duchene M, Aspoeck H, Walochnik J 2008. Acanthamoeba strains lose their abilities to encyst synchronously upon prolonged axenic culture. Parasitol Res 102: 1069-1072.

Magliano AC, Silva FM, Teixeira MM, Alfieri SC 2009. Genotyping, physiological features and proteolytic activities of potentially pathogenic Acanthamoeba sp. isolated from tap water in Brazil. Exp Parasitol 123: 231-235.

Marciano-Cabral F, Cabral G 2003. Acanthamoeba spp as agents of disease in humans. Clin Microbiol Rev 16: 273-307.

Martinez AJ 1985. Free-living amebas: natural history, prevention, diagnosis, pathology and treatment of disease, CRC Press, Boca Raton, $156 \mathrm{pp}$.

Martinez AJ 1991. Infections of the central nervous system due to Acanthamoeba. Rev Infect Dis 13: 399-402.

Martinez AJ, Visvesvara GS 1997. Free-living, amphizoic and opportunistic amebas. Brain Pathol 7: 583-598.

Mazur T, Gustawska L, Hadás E 1999. Extracerebral infections of Acanthamoeba spp in mice. Pathomorphological changes in tissues of infected animals. Wiad Parazytol 45: 83-87.

Mazur T, Hadas E 1994. The effect of the passages of Acanthamoeba strains through mice tissues on their virulence and its biochemical markers. Parasitol Res 80: 431-434.

Mazur T, Jóźwiak M 1993. Extracerebral infections of Acanthamoeba spp in mice. Wiad Parazytol 39: 357-366.

Moreira D, Santarém N, Loureiro I, Tavares J, Silva AM, Amorim AM, Ouaissi A, Cordeiro-da-Silva A, Silvestre R 2012. Impact of continuous axenic cultivation in Leishmania infantum virulence. PLoS Negl Trop Dis 6: 1460-1469.

Mortazavi PN, Goldsworthy G, Kirk R, Khan NA 2009. Novel model for the in vivo study of central nervous system infection due to Acanthamoeba spp (T4 genotype). J Med Microbiol 58: 503-508.

Santos AL, Santos DO, Freitas CC, Ferreira BLA, Afonso IF, Rodrigues CR, Castro HC 2007. Staphylococcus aureus: visiting a strain of clinical importance. J Bras Patol Med Lab 43: 413-423.

Stevens AR, O'Dell WD 1974. In vitro growth and virulence of Acanthamoeba. J Parasitol 60: 884-885.

Young AL, Leboeuf NR, Tsiouris SJ, Husain S, Grossman ME 2010. Fatal disseminated Acanthamoeba infection in a liver transplant recipient immunocompromised by combination therapies for graft-versus-host disease. Transpl Infect Dis 12: 529-537. 